\title{
Staatlich verordnete Prävention
}

$\mathrm{E}$ s ist sattsam bekannt: Unser Gesundheitswesen ist moribund. Der Patient ist am Ende. Seine Ärzte stehen um ihn herum, lamentieren, analysieren, schlagen vor, diskutieren, schieben sich gegenseitig den schwarzen Peter zu - und sind ratlos. Just da fühlt sich die Politik berufen, den Karren aus dem Dreck zu ziehen, dem Ungemach mit Gesetzen Einhalt zu gebieten und unser Gesundheitswesen in eine neue, bessere Zukunft zu führen. Gesundheitspolitiker aller Couleur haben sich inzwischen so manches einfallen lassen: Disease-Management-Programme (DMPs), Diagnosis Related Groups (DRGs), ja ein komplettes Gesundheitsmodernisierungsgesetz. Und weil das die Kosten wohl doch nicht ausreichend senkt, setzt man nun auf Prävention. Der Joker!

Wir Ärzte wissen schon lange, dass man da nicht länger zuschauen kann. Die Deutschen rauchen weiter, lassen es sich schmecken und laufen höchstens in ihrer Fantasie vor dem Fernsehgerät. Allen modischen Fitnesstrends und Trendsportaktivitäten zum Trotz - die Deutschen bewegen sich höchst ungern. 35\% der Bevölkerung rauchen nach wie vor (das sind 30\% mehr als im europäischen Durchschnitt!). Über 70\% der Männer und Frauen im Alter über 60 Jahre haben erhöhte Cholesterinwerte, 21\% der Frauen und 18\% der Männer im Alter zwischen 25 und 69 Jahren sind übergewichtig. Rund $8 \%$ der Erwachsenen leiden unter Typ-2-Diabetes, das sind sieben Millionen Menschen in Deutschland. Hochgerechnet werden es in nur fünf Jahren gut zehn Millionen sein. Eine viel zitierte Pikanterie: Der jüngste Typ-2-Diabetiker in Deutschland ist sieben Jahre alt.

Also Prävention - eine viel versprechende ganz neue Strategie: den Leuten klar zu machen, wie sie ihr Leben umzustellen haben, um künftig gesünder, leistungsfähiger, reparaturresistenter zu sein. Dass sich eine solch hehre Absicht nicht ganz so elegant realisieren lässt, ist uns allen klar. Ein Gesetz soll jetzt Abhilfe schaffen, das Präventionsgesetz § 20 SGB V. Petra Selg, das grüne Parlamentsmitglied, gibt sich euphorisch: „Wir möchten der Prävention neben der Gesundheit, der Rehabilitation und der Pflege einen eigenständigen Stellenwert geben.“ Beifall allerorts, denn gegen Prävention hat keiner etwas einzuwenden. Das ist gut und sinnvoll.

Doch ein Gesetz muss umgesetzt und beaufsichtigt werden, Gelder und Aktivitäten sind zu koordinieren. Das schafft Arbeitsplätze in der Verwaltung und dem Controlling. Aber wer soll das bezahlen? Bund und Länder lamentieren über leere Kassen. Also müssen die Krankenkassen herhalten. Die sollen von ihrem ohnehin schmächtigen Obolus von 2,70 Euro, den sie rechnerisch für jedes Mitglied bislang für präventive Maßnahmen auszugeben gehalten sind, noch ein Teilchen abzwacken und dem zentralen Präventionstopf überweisen. Das ist nichts weiter als ein heimtückischer Finanztransfer aus dem kurativen Topf in den präventiven. Wohl als Vorgriff auf Einsparungen im kurativen Sektor, wenn die Prävention denn einmal greifen sollte. Ein frommer Wunsch, denn Prävention braucht viel Zeit, und wenn es funktioniert, merken wir erst in Jahren, dass sich dadurch etwas einsparen lässt. Einstweilen fehlt dann aber Geld für Diagnose und Therapie. Statt Sartanen verschreiben wir den Hypertonikern dann, ganz im Sinne von Herrn Lauterbach, preiswertere Diuretika.

Übrigens haben die Krankenkassen zum Teil aus ihrem Präventionstopf schon hin und wieder durchaus interessante regionale Präventionsmaßnahmen realisiert - denn Präventionsstrategien lassen sich nicht zentral umsetzen. Das ist mühsame Kleinstarbeit, vor Ort, mit intimer Kenntnis des Klientel. Dafür werden die Kassen nun künftig weniger Geld zur Verfügung haben. Dafür kassiert die Präventionsstiftungsbehörde bundesweit die Gelder, verwaltet sie, lässt groß angelegte Werbekampagnen entwickeln. Das Resultat? Das Geld ist weg, und die Deutschen rauchen weiter, werden dicker, werden kränker.

Prävention funktioniert nicht per Dekret. Sie erfordert ungeheure Geduld, die berühmte Arbeit an der Basis. Permanente Aufklärung vom Kindergarten an auch gegen die aggressiven Werbestrategien der Süßwaren- und Fast-Food-Industrie. Wir Ärzte müssen dabei an vorderster Front mitwirken. Wir haben die Kompetenz und könnten in die Schulen gehen, wenn man uns nur ließe. Wir müssen unsere Autorität dafür einsetzen. Es wäre schade, wenn wir das neue bürokratische Unheil namens Präventionsgesetz einfach widerspruchslos über uns ergehen lassen und wider besseres Wissen hoffen, dass schon ein Wunder geschehen wird. Verlassen wir uns nicht darauf. Nutzen wir wenigstens die Tatsache, dass dieses Gesetz den Begriff der Prävention attraktiver gemacht hat. Versuchen wir, jeder in seinem Umfeld und mit hohem persönlichen Engagement, konkret aufzuklären, für ein Quäntchen mehr an Gesundheit zu werben und unsere Patienten zu Partnern in Sachen Gesundbleiben zu machen.

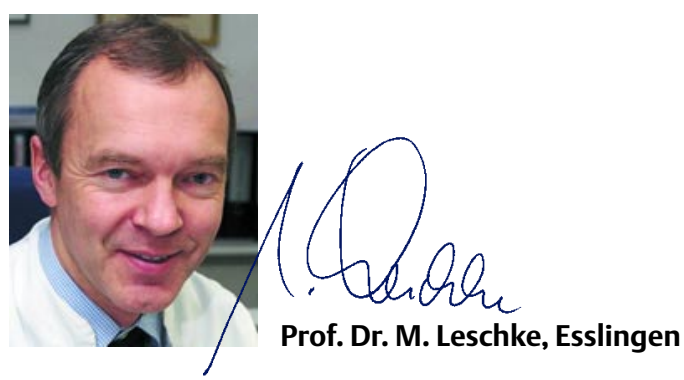

